\title{
Li and Be Depletion in Stars with Exoplanets?
}

\section{E. Delgado Mena ${ }^{1,2}$, G. Israelian ${ }^{1,2}$, J. I. González Hernández ${ }^{1,2}$, R. Rebolo ${ }^{1,2,3}$, N. C. Santos ${ }^{4,5}$, S. G. Sousa ${ }^{4,5}$, and J. Fernandes ${ }^{6}$}

${ }^{1}$ Instituto de Astrofísica de Canarias, E-38200 La Laguna, Tenerife, Spain. email: edm@iac.es

${ }^{2}$ Departamento de Astrofísica, Universidad de La Laguna, 38205 La Laguna, Tenerife, Spain.

${ }^{3}$ Consejo Superior de Investigaciones Científicas, 28006, Madrid, Spain

${ }^{4}$ Centro de Astrofísica, Universidade do Porto, Rua das Estrelas, 4150-762 Porto, Portugal.

${ }^{5}$ Departamento de Física e Astronomia, Faculdade de Ciências, Universidade do Porto, Portugal.

${ }^{6}$ Centro de Física Computacional, Universidade de Coimbra, Portugal.

${ }^{7}$ Observatório Astronómico e Departamento de Matemática, Universidade de Coimbra, Portugal.

\begin{abstract}
It is well known that stars with orbiting giant planets have a higher metallic content than stars without detected planets. In addition, we have found that solar-type stars with planets present an extra Li depletion when compared with field stars. On the other hand, Be needs a greater temperature to be destroyed, so we may find such a relation in cooler stars, whose convective envelopes are deep enough to carry material to layers where Be can be burned. We present $\mathrm{Li}$ and Be abundances for an extensive sample of stars with and without detected planets, covering a wide range of effective temperatures $(4700-6500 \mathrm{~K})$ with the aim of studying possible differences between the abundances of both groups. The processes that take place in the formation of planetary systems may affect the mixing of material inside their host stars and hence the abundances of light elements.
\end{abstract}

Keywords. stars: abundances — stars: atmospheres — stars: fundamental parameters — stars: planetary systems - stars: planetary systems: formation

\section{Lithium}

In a previous work, we reported an extra Li depletion in planet-host solar-type stars when compared with stars without planets (Israelian et al. 2009). Here, we present new Li abundances for the HARPS metal-poor sample of stars with and without planets (Santos et al. 2011, Sousa et al. 2011). In the left panel of Figure 1, these new Li abundances are plotted with those of the stars in the HARPS GTO sample and some planet hosts from other surveys, as a function of age. Lithium is expected to decrease with age, therefore, if planet host stars were on average older than comparison sample stars they would have depleted more lithium. However, we can see that comparison sample stars are still equally distributed at both sides of $\log \mathrm{N}(\mathrm{Li})=1.5$. Moreover, age does not seem to play a role in this behaviour (Sousa et al. 2010), except for the younger objects (age $<2$ Gyr). Indeed, $50 \%$ of comparison stars present high $\mathrm{Li}$ abundances for a wide range of ages, although most of the planet-hosts show a severe Li destruction regardless of their age.

We propose that the low $\mathrm{Li}$ abundance of planet-host solar-analogue stars is directly associated with the presence of planets due to the effect of these in the angular momentum evolution of the star and the surface convective mixing (Bouvier 2008, Israelian et al. 

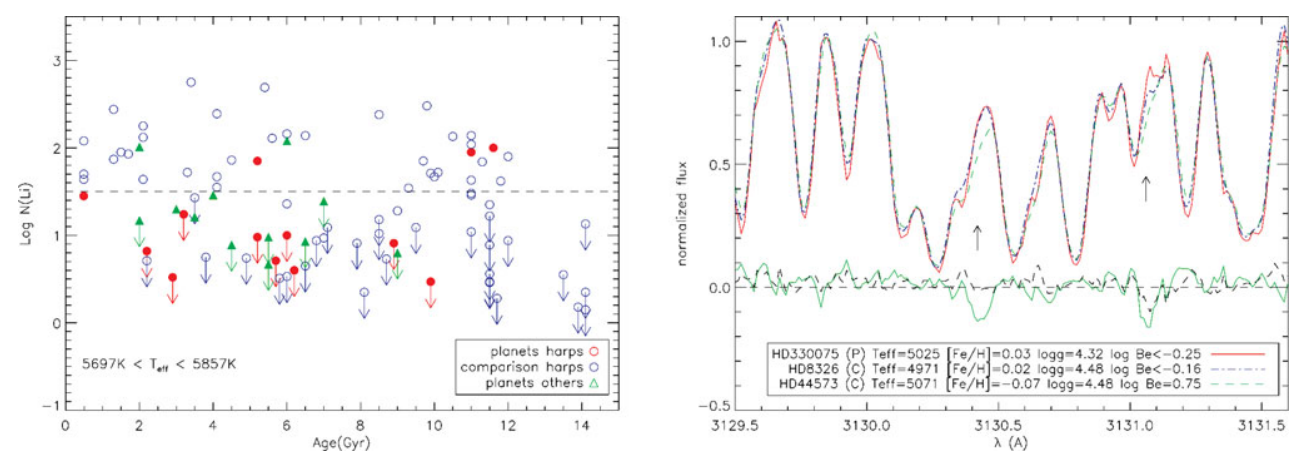

Figure 1. Left panel: Li abundances as a function of age for solar analogue stars with (red filled circles) and without (blue open circles) planets from HARPS GTO and HARPS metal-poor samples. Green triangles are stars with planets from other surveys. Right panel: Real spectra around Be II lines (located at the arrows) for the planet host HD330075 (red line) and the comparison stars HD8326 (blue dashed line). The black and green lines represent the differences in flux between spectra.

2004). To understand this Li extra depletion in stars with planets, observations of younger stars and proper modelling are required.

\section{Beryllium}

The determination of Be abundances for the coolest stars $\left(\mathrm{T}_{\text {eff }}<5200 \mathrm{~K}\right)$ is very difficult since the Be II line at $3131.06 \AA$ is blended with a Mn I line which becomes stronger as the temperature decreases, therefore, in this $\mathrm{T}_{\text {eff }}$ regime the uncertainties in Be abundances are large. To determine possible differences between stars with and without planets, we propose to compare directly the spectra of stars with very similar parameters, $\mathrm{T}_{\text {eff }},[\mathrm{Fe} / \mathrm{H}], \log \mathrm{g}, \mathrm{v} \sin i$, and $\mathrm{Mn}$ abundance. Therefore, if there is a difference between the spectra of two stars, it should be due to a difference in Be abundance. In a previous study (Delgado Mena et al. 2011), we found two planet hosts which showed a clear depletion of Be when compared to several stars without planets. One of them, HD330075, is shown in the right panel of Figure 1 together with two new comparison stars. HD44573 shows stronger Be lines than the planet host although HD8326 shows a similar level of depletion. In this work, we analyze more pairs of analogue cool stars but all of them present weak Be lines and no differences are observed between their fluxes. Therefore, the effect of extra Li depletion in solar-type stars with planets when compared with stars without detected planets does not seem to be present for Be, although the number of cool stars is still too small to reach a final conclusion.

\section{References}

Bouvier, J. 2008, A\& A, 489, 53

Israelian, G., Santos, N. C., Mayor, M., \& Rebolo, R. 2004, A\&A, 414, 601

Delgado Mena, E., Israelian, G., González Hernández J. I., Santos, N. C., \& Rebolo. R. 2011, ApJ, 728, 148

Israelian, G., Delgado Mena, E. Santos, N. C., Sousa, S. G., Mayor, M., Udry, S., Domínguez Cerdeña, C., Rebolo, R., \& Randich, S. 2009, Nature, 462, 189

Santos, N. C., et al., 2011, A\&\&A, 526, A112

Sousa, S. G., Fernandes, J., Israelian, G., \& Santos, N. C. 2010, A\&\&A, 512, L5

Sousa, S. G., Santos, N. C., Israelian, G., Lovis, C., Mayor, M., Silva, P. B., \& Udry, S. 2011, $A \mathscr{G} A, 526, \mathrm{~A} 99$ 